\title{
Wideband High-Gain and Low Scattering Antenna Using Shared-Aperture Metamaterial Superstrate
}

\author{
Chen ZHANG, Xiang-yu CAO, Jun GAO, Si-jia LI \\ Information and Navigation Institute of Air Force Engineering University, Xi'an, Shaanxi, 710077, China \\ xue320long@sina.cn, xiangyucaokdy@163.com,gjgj9694@163.com,1sj051@126.com
}

Submitted September 29, 2017 / Accepted December 27, 2017

\begin{abstract}
In this paper, a novel wideband high-gain and low scattering antenna using shared-aperture metamaterial superstrate (SAMS) is designed, fabricated and measured. The superstrate unit cell consisting of two frequency selective surface (FSS) layers with a positive reflection phase gradient is designed to enhance the antenna gain. Then, three different sizes of single units are arranged as a shared-aperture configuration to form the metamaterial superstrate, which is loaded onto the antenna. By utilizing the phase compensation property along different units, the antenna gain enhancement bandwidth is effectively broadened. By adjusting the SAMS loading height, the antenna radar cross section (RCS) is also reduced obviously owing to the different reflective wave phases of PRS and antenna ground. After loading SAMS, the antenna possesses an impedance bandwidth of $44.7 \%$ from $7.8 \mathrm{GHz}$ to $12.3 \mathrm{GHz}$, covering the whole X band. From $7.9 \mathrm{GHz}$ to $12.1 \mathrm{GHz}$, the antenna has an obvious gain enhancement, with a peak of $7 \mathrm{~dB}$, meanwhile, the antenna RCS is effectively reduced from $4 \mathrm{GHz}$ to $12 \mathrm{GHz}$ and the maximum $\mathrm{RCS}$ reduction reaches $25.4 \mathrm{~dB}$ at $8.6 \mathrm{GHz}$ for x-polarized incident wave and $15.8 \mathrm{~dB}$ for $y$-polarized incident wave. The results are validated by both numerical simulation and experimental measurements.
\end{abstract}

\section{Keywords}

Wideband, gain enhancement, RCS reduction, sharedaperture, Fabry-Perot resonator

\section{Introduction}

In recent years, with the development of antenna technology, the requirements of antenna properties are becoming more and more strictly. To be specific, there are two main parts, radiation performance and scattering performance. On one hand, such as satellite communication and navigation systems demand, antennas should have wideband and high directivity [1], on the other hand, the radar cross section (RCS) should be reduced owing to military requirements [2], [3]. Traditional ways to enhance the antenna gain are mainly divided into two ways, changing the antenna structure and adopting antenna arrays [4], [5], which would suffer from design complexity, high cost, power losses in the feeding network and such similar problems, and these will also result in the raise of the target RCS which is harmful to their stealth properties. Therefore, the realization of wideband high gain and low scattering antenna has been a major problem [6].

Metamaterial usually refers to a kind of artificial periodic structures [7], it has physical properties that are not possessed by natural material. In recent years, metamaterial has received extensive attention owing to its exotic electromagnetic properties [8]. For instance, [9] designed a horn antenna with metamaterial lens to enhance the antenna gain [10] proposed broadband aperture antennas using intrinsic dispersion properties of metamaterial. Placing one or more layers of metamaterial superstrate in front of a simple antenna will achieve high gain Fabry-Perot (FP) resonator antenna (FPRA), at present, the superstrate is at a proper distance from a ground plane, creating an air-filled cavity between the partially reflective surface (PRS) and the ground plane. After one or more reflections in the cavity, the electromagnetic wave of antenna radiation will stack up with the direct transmission wave, thus, the gain of the antenna is improved [11]. When meeting the following formula, the directivity of the antenna achieves the maximum [12],

$$
h_{\mathrm{r}}=\frac{c}{4 \pi f}\left(\varphi_{\mathrm{PRS}}+\varphi_{\mathrm{GND}}-2 N \pi\right), \quad N=0,1,2, \cdots
$$

where $\varphi_{\mathrm{PRS}}$ and $\varphi_{\mathrm{GND}}$ are the reflection phases of PRS and the ground plane, $f$ is the operating frequency of the antenna and $h_{\mathrm{r}}$ is the distance between the PRS and the ground plane. Meanwhile, when antenna is irradiated as a scatterer, the electromagnetic waves reflected by PRS and metal ground will cancel with each other owing to the phase difference and RCS will decrease obviously.

FP resonator was initially used in the field of optics, in 1956, G. V. Trentini firstly placed the superstrate structure possessing partially reflective property in front of a waveguide horn and the antenna gain enhancement was realized [13]. Later, FPRA was widely studied, FPRA is a kind of highly directive antenna with high radiation efficiency and low complexity compared to conventional 
antennas, however, there are few reports about the scattering performance of FPRA. In 2015, Zheng utilized two $180^{\circ}$ phase difference artificial magnetic conductor (AMC) cells to combine a chessboard configuration superstrate [14]. In 2017, Zhang combined the coding metasurface and FPRA together to reduce the antenna RCS and keep high gain [15]. More high-gain and low-RCS FPRA can be found in [16]. Nevertheless, these FPRAs can only worked in narrow bandwidths and is not suitable for many wideband antenna applications.

In this paper, a wideband high-gain and low scattering antenna using shared-aperture metamaterial superstrate (SAMS) is designed and fabricated. The PRS is combined of three different sizes of two-layer frequency selective surfaces (FSSs). For radiation performance, the sharedaperture configuration utilizes the phase compensation property along different FSSs, broadening the gain enhancement bandwidth effectively. For scattering performance, antenna RCS is obviously reduced owing to the phase cancellation of different reflected waves. After loading SAMS, the antenna possesses an impedance bandwidth of $44.7 \%$ for the reflection coefficient $S_{11}$ below $-10 \mathrm{~dB}$ from $7.8 \mathrm{GHz}$ to $12.3 \mathrm{GHz}$. From $7.9 \mathrm{GHz}$ to $12.1 \mathrm{GHz}$, the antenna has an obvious gain enhancement, with a peak of $7 \mathrm{~dB}$, meanwhile, the antenna RCS is effectively reduced from $4 \mathrm{GHz}$ to $12 \mathrm{GHz}$. Simulated and measured results verify the correctness of this antenna design method.

\section{Analysis and Design of FPRA}

\subsection{Design of SAMS}

The geometry of the superstrate unit cell is illustrated in Fig. 1. We present the structure with two different FSS layers. Two different patches are printed on the substrate with a relative permittivity of 2.65 . The metal pattern on the top of the unit is a square patch, and on the bottom the metal pattern is a cross patch. The square FSS has the property of low-pass filter and the cross FSS has the property of high-pass filter, therefore, this two-layer FSSs should resonate in a wide frequency range. The parameters of the unit are as follows: $a=4.9 \mathrm{~mm}, t=1 \mathrm{~mm}, P=5.9 \mathrm{~mm}$ and $w=0.9 \mathrm{~mm}$. In order to explain the wideband property more clearly, equivalent circuit is used, as shown in Fig. 2. For the metal pattern on the top of the unit, each horizontal gap between two square patches acts as a capacitor, whose capacitance $C_{2}$ can be easily controlled by changing the width and length of the gap, namely, changing the size of the patch. In addition, the patch itself also provides an inductance with a small value $L_{2}$, connected in series with the capacitor. For the metal pattern on the bottom of the unit, the cross patch can act as an inductance $L_{1}$, the large separation between each cross patch acts as a small capacitance $C_{1}$, which is parallel with the inductors. Owing to the small value of $C_{1}$ and $L_{2}$, it is worth nothing that neither the square patch nor the cross patch can produce a resonance, therefore, in a wideband frequency range, the superstrate unit can behave good impedance characteristic.

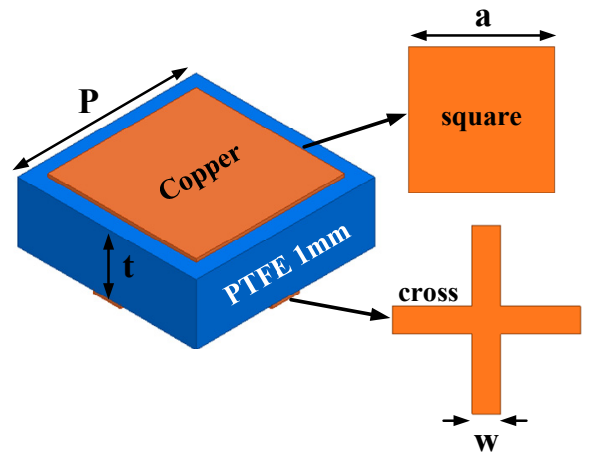

Fig. 1. Geometry of the superstrate unit cell.

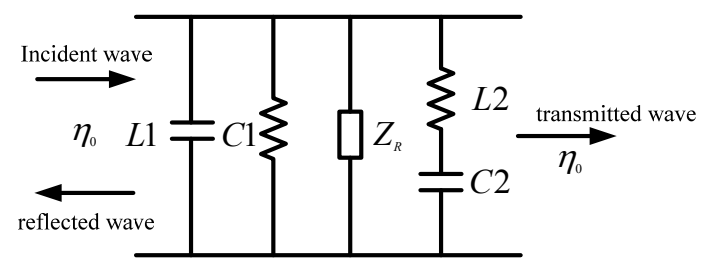

Fig. 2. Equivalent circuit of the unit cell.

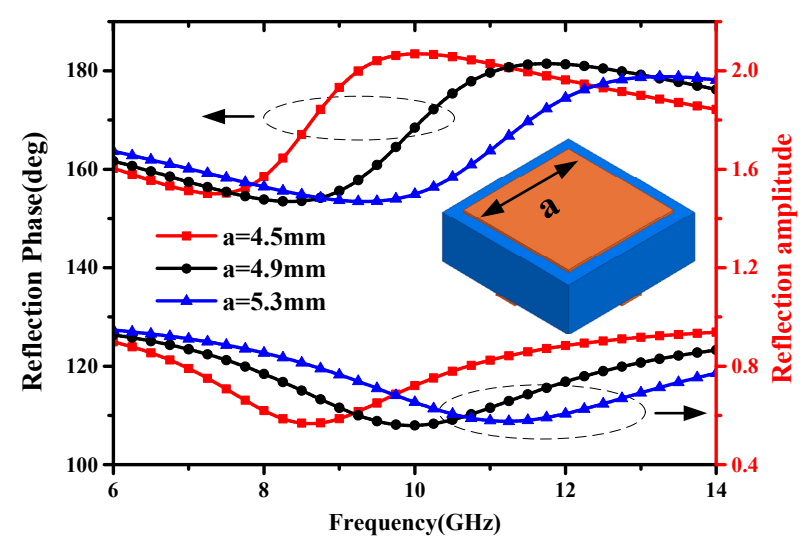

Fig. 3. Reflection phase and amplitude of the superstrate unit cell when $a$ changes.

Figure 3 shows the simulated results of the reflection phase and amplitude of the superstrate unit when the square patch size $a$ changes. When $a=4.9 \mathrm{~mm}$, the corresponding reflection phase of the unit increases at the frequency from $8.3 \mathrm{GHz}$ to $11.8 \mathrm{GHz}$, having the positive phase gradient. The reflection magnitude in the whole frequency band is above 0.56 . According to (1), the proposed structure should have the potential to enhance the antenna gain in a wide frequency range.

However, the gain enhancement bandwidth still could not cover the whole X band, it should be further extended. In recent years, shared aperture technique has become more and more mature in antenna array domain. This technique is usually applied to extend the array bandwidth, inspired by this, we hope the superstrate bandwidth could also be extended in this way. Simulated results indicate that when parameter $a$ changes, the superstrate unit cell has different reflection phases but the same coefficient, therefore, if these three different sizes of units combine as a sharedaperture structure, utilizing the phase compensation property, the configuration should broaden the gain enhance- 


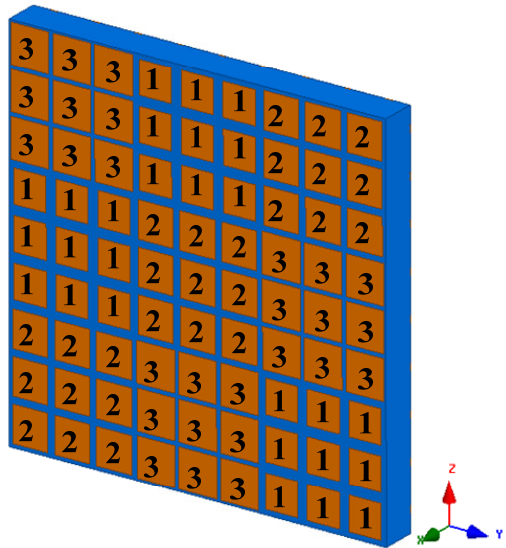

Fig. 4. Geometry of SAMS.

ment bandwidth effectively. To verify the feasibility of this method, three units are numbered as ' 1 ' ' 2 ' ' and ' 3 ' when parameter $a=4.5 \mathrm{~mm}, 4.9 \mathrm{~mm}$ and $5.3 \mathrm{~mm}$, respectively. Considering the periodicity properties of each unit, superstrate unit block arrays have been designed, each block is formed by 9 ' 1 ', ' 2 ' or ' 3 ' elements. The final SAMS consists of $3 \times 3$ block arrays as a chessboard configuration, shown in Fig. 4.

\subsection{Design of FPRA Using SAMS}

As an integral part of FP resonator antenna, the feeding antenna also plays an important role. In this design, a novel magneto-electric antenna is chosen for its low profile and wide bandwidth. The whole antenna uses a dielectric slab with a thickness of $3 \mathrm{~mm}, \varepsilon_{\mathrm{r}}=4.4$ and $\tan \delta=0.02$. Electric dipole metal patches and magnetic dipole metal vias are excited by a T-shaped feeding line. The dimensions of the antenna are listed as follows: $b=5.6 \mathrm{~mm}$, $c=2 \mathrm{~mm}, d=5 \mathrm{~mm}, L=7 \mathrm{~mm}, m=2.3 \mathrm{~mm}, n=3.8 \mathrm{~mm}$ and $s=2.2 \mathrm{~mm}$. The geometry of the whole FPRA is shown in Fig. 5.

\section{Analysis of FPRA Using SAMS}

\subsection{Radiation Properties}

Figure 6 shows the comparisons of simulated reflection coefficient $S_{11}$ and gain with and without SAMS.

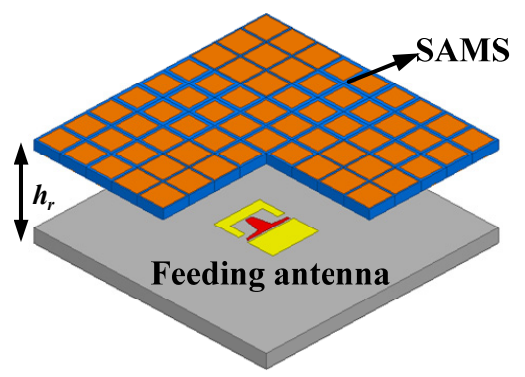

(a)

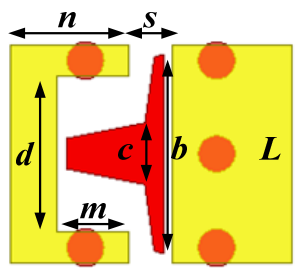

(b)
Fig. 5. Geometry of (a) the whole FP resonator antenna and (b) the feeding antenna.

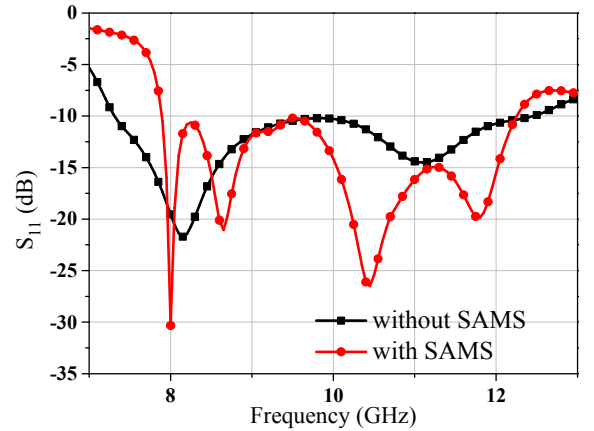

(a)

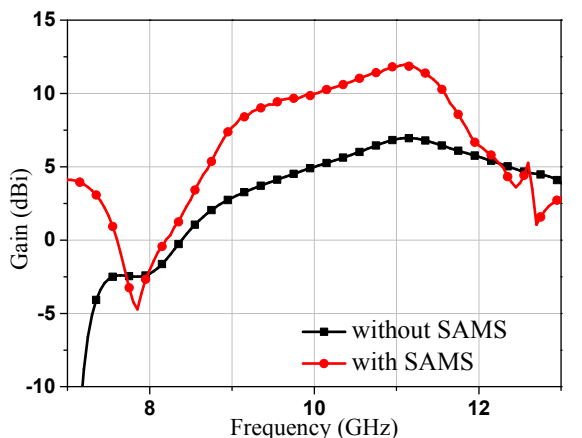

(b)

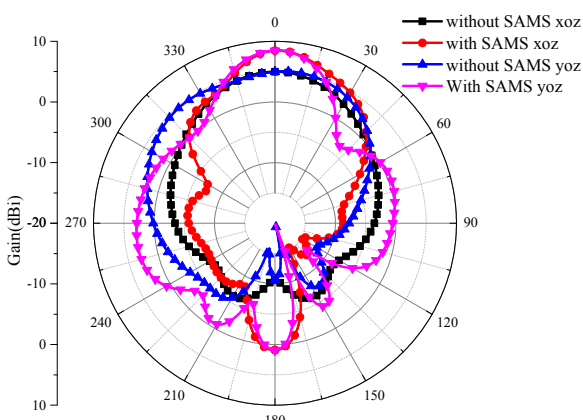

(c)

Fig. 6. Comparisons of simulated results with and without SAMS: (a) reflection coefficient $S_{11}$, (b) antenna gain, (c) radiation patterns at $10 \mathrm{GHz}$.

It can be seen the original antenna working bandwidth ranges from $7.3 \mathrm{GHz}$ to $12.5 \mathrm{GHz}$, after loading SAMS, the bandwidth only decreases a little, ranging from $7.8 \mathrm{GHz}$ to $12.3 \mathrm{GHz}$; For the antenna gain, a relative gain enhancement bandwidth has reached to $42 \%$ from $7.9 \mathrm{GHz}$ to $12.1 \mathrm{GHz}$ with a maximum gain enhancement of $7 \mathrm{~dB}$. The superstrate unit with a positive phase gradient is only ranging from $8.3 \mathrm{GHz}$ to $11.8 \mathrm{GHz}$, after adopting SAMS, the gain enhancement bandwidth is effectively broadened, covering the whole $\mathrm{X}$ band. Figure 6(c) shows the radiation pattern at $10 \mathrm{GHz}$. It can be seen that comparing with the original antenna, after loading SAMS, the HPBW in xoz plane has been decreased from $72^{\circ}$ to $41^{\circ}$, and from $115^{\circ}$ to $37^{\circ}$ in yoz plane. The directivity of antenna has been well enhanced and the antenna has a low side-lobe level. The beam peak direction is towards the front side direction and the maximum gain has increased $5 \mathrm{~dB}$ around. By comparison, we can see the SAMS converges the radiated electromagnetic wave to the normal direction and the gain enhancement effect is achieved. The efficiency of the antenna with SAMS is also calculated, at $10 \mathrm{GHz}$, the directivity of 
the whole antenna is $8.88 \mathrm{dBi}$ around, for an antenna with a certain directivity, the structure efficiency can be estimated by the following equation [18]

$$
A=\frac{10^{D / 10} \cdot \lambda^{2}}{E \cdot \pi^{2}}
$$

where $A$ is the size of the square surface of the antenna, $\lambda$ is the wavelength, $E$ is the efficiency and $D$ is the directivity of the antenna. By calculation, the efficiency in normal direction is $26 \%$ around, due to the widebeam property, the efficiency is not very high.

Generally, there are several other types of SAMS configurations by using three different sizes of units, for example, helix configuration and fence-like configuration, as shown in Fig. 7(a) and 7(b). These two configurations are selected in our consideration for comparing their gain enhancement abilities, they are simulated and analyzed respectively.

For the helix configuration, the spiral center is element ' 1 ', expanding from inside to outside, each different size consists of 27 elements. Comparing with the original SAMS analyzed above, this configuration will result in degradation of gain enhancement bandwidth, the gain of the antenna is enhanced only from $8.1 \mathrm{GHz}$ to $9.6 \mathrm{GHz}$, not covering the whole $\mathrm{X}$ band, shown in Fig. 8. According to analysis, in this configuration, only elements ' 1 ' possess periodic property, the other two sizes of units arrange dispersedly and do not behave it, therefore, this kind of SAMS cannot broaden the antenna gain enhancement bandwidth.

For the fence-like configuration, using this kind of SAMS will broaden the antenna gain enhancement bandwidth comparing with using single units superstrate. From $8.1 \mathrm{GHz}$ to $12.1 \mathrm{GHz}$, the antenna gain is enhanced, but this configuration will result in severe antenna beam deflection, the antenna main beam is not in the normal direction. This is because the fence-like SAMS configuration resulted in the inhomogeneous phase distribution. As shown in Fig. 9, the antenna beam in $\mathrm{H}$ plane deflects severely.

Through the above analysis, designing SAMS configuration to broaden antenna gain enhancement should meet the following two conditions. One is because single superstrate unit does not have the corresponding reflection characteristic in application, at least $3 \times 3$ units combined together can satisfy the condition. The other is that the three sizes of unit block arrays should be arranged as uniformly as possible. Therefore, the original SAMS configuration is a relatively ideal arrangement.
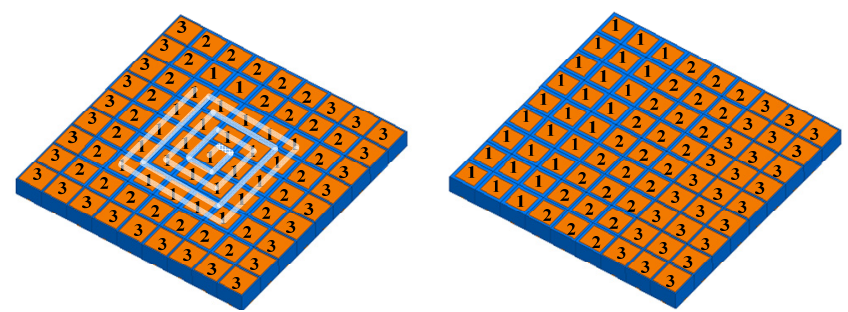

Fig. 7. Other two SAMS: (a) helix configuration, (b) fencelike configuration.

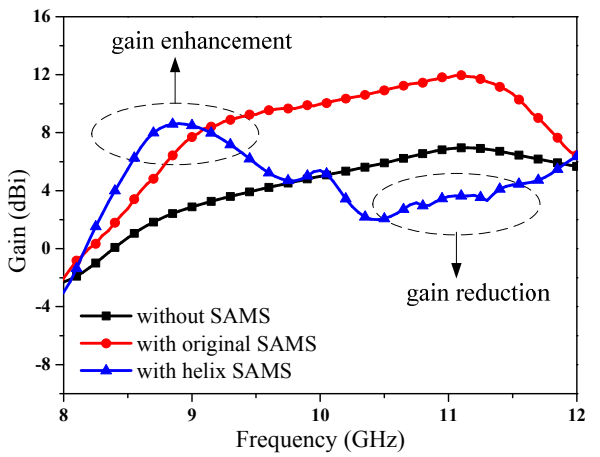

Fig. 8. Simulated antenna gain with and without SAMS.

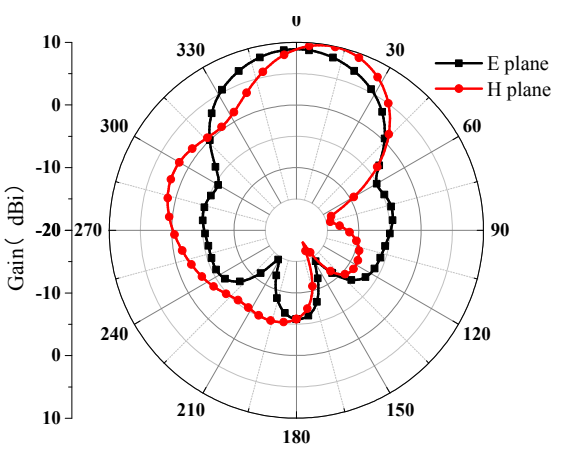

Fig. 9. Fence-like SAMS antenna radiation pattern at $10 \mathrm{GHz}$.

\begin{tabular}{|l|c|c|c|c|}
\hline & {$[17]$} & {$[18]$} & {$[19]$} & This paper \\
\hline Antenna size $(\lambda)$ & $4.5 \lambda \times 4.5 \lambda$ & $2.4 \lambda \times 2.4 \lambda$ & $6 \lambda \times 6 \lambda$ & $1.8 \lambda \times 1.8 \lambda$ \\
\hline $\begin{array}{l}\text { Antenna bandwidth } \\
(\%)\end{array}$ & 15.6 & 28 & 13.2 & 44.7 \\
\hline Peak gain (dBi) & 16.2 & 13.8 & 22.7 & 12.2 \\
\hline $\begin{array}{l}\text { Maximum gain } \\
\text { enhancement }(\mathrm{dB})\end{array}$ & & 7.8 & 5.5 & 7 \\
\hline
\end{tabular}

Tab. 1. Comparison among four antennas.

Table 1 shows a comparison between the proposed design and existing designs in antenna radiation performance. It can be seen that comparing with traditional FP antenna, the superiority in this paper is the SAMS gain enhancement bandwidth which is relatively wider providing a new way to broaden the antenna gain enhancement bandwidth. Certainly, there are also some shortcomings to be solved, compared with [18], the antenna efficiency in normal direction is not very high, this is because the antenna itself is a kind of widebeam antenna and the antenna aperture is relative small, besides, the antenna structure in this paper is more complex compared with [17]. These are problems that need to be improved in the future.

\subsection{Scattering Properties}

In order to realize the low scattering properties, the loading height of the superstrate has an important impact on the FPRA. The principle of reducing the antenna RCS is shown in the following figure. When electromagnetic wave irradiate FPRA, the reflected wave of PRS and the antenna will have a phase difference, through phase cancellation principle, the antenna RCS will have an obvious reduction. 


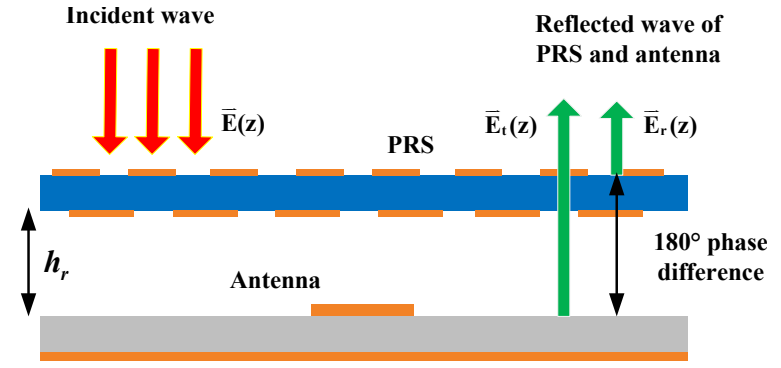

Fig. 10. Scattering schematic model of FPRA.

In the figure above, $E(z), E_{\mathrm{r}}(z)$, and $E_{\mathrm{t}}(z)$ are amplitudes of incident wave, reflected waves of PRS and antenna, their expressions are:

$$
\begin{gathered}
\boldsymbol{E}_{\mathrm{r}}(z)=E_{\mathrm{r}} \exp \left[\mathrm{j}\left(-k z+\varphi_{\mathrm{r}}\right)\right], \\
\boldsymbol{E}_{\mathrm{t}}(z)=E_{\mathrm{t}} \exp \left[\mathrm{j}\left(k z+\varphi_{\mathrm{t}}\right)\right]
\end{gathered}
$$

where $\varphi_{\mathrm{r}}$ and $\varphi_{\mathrm{t}}$ are phases of the waves, the whole scattering field of FPRA is

$$
\boldsymbol{E}(z)=\boldsymbol{E}_{\mathrm{r}}(z)+\boldsymbol{E}_{\mathrm{t}}(z) .
$$

Through the above analysis, when adjusting loading height $h_{\mathrm{r}}$, the antenna RCS will be reduced, meanwhile, according to (1), $h_{\mathrm{r}}$ has an important impact on the gain enhancement of FPRA, therefore, if $h_{\mathrm{r}}$ takes a suitable value, both antenna radiation and scattering performance will be improved. After optimizing the parameter, the height is finally selected as $h_{\mathrm{r}}=15.5 \mathrm{~mm}$.

In order to validate the RCS reduction of the proposed FPRA, the monostatic RCS for both x-polarized and y-polarized incident waves impinging from normal direction have been simulated, shown in Fig. 11.

To validate the broadband low RCS characteristic, a reference antenna without SAMS is used for comparison. The monostatic RCS of both the antennas for x-polarized and $y$-polarized incident waves impinging from normal direction have been simulated, shown in Fig. 11. As predicted, owing to phase cancellation, the proposed FPRA has a broadband RCS reduction from $4 \mathrm{GHz}$ to $12 \mathrm{GHz}$, the maximum RCS reduction reaches $25.4 \mathrm{~dB}$ at $8.6 \mathrm{GHz}$ for $\mathrm{x}$-polarized incident wave and $15.8 \mathrm{~dB}$ for $\mathrm{y}$-polarized incident wave. Also, the bistatic RCS at $8.6 \mathrm{GHz}$ is simulated. Figure 12(a) and (b) shows the simulated 2-D bistatic RCS patterns for x-polarized wave at Xoz and yoz planes. It can be seen that antenna RCS is significantly reduced at the elevation angles between $-30^{\circ}$ to $+30^{\circ}$. Owing to phase cancellation of PRS and antenna, the reflected waves emerge diffuse scattering phenomenon. Therefore, compared with the reference antenna, RCS is increased at other angle ranges and FPRA has two different 2-D bistatic RCS patterns. Figure 12(c) and (d) shows 3-D bistatic RCS patterns for the proposed FPRA and the reference antenna, respectively. It is obvious that the RCS is reduced after loading SAMS. The simulated results certify the accuracy of the antenna design.

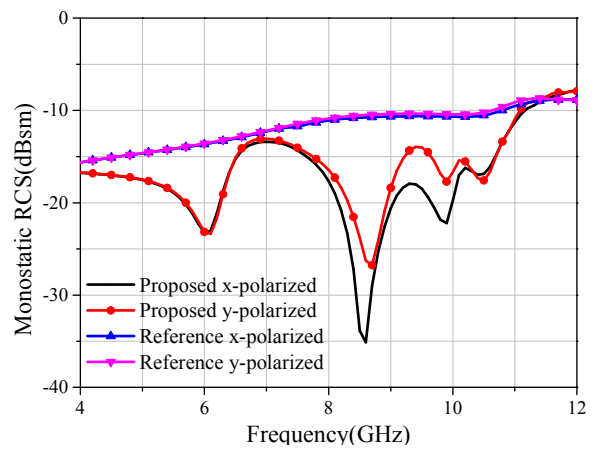

Fig. 11. Simulated results of monostatic RCS.

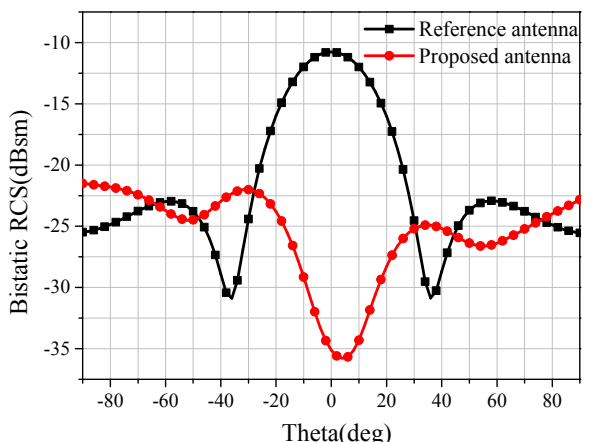

(a)

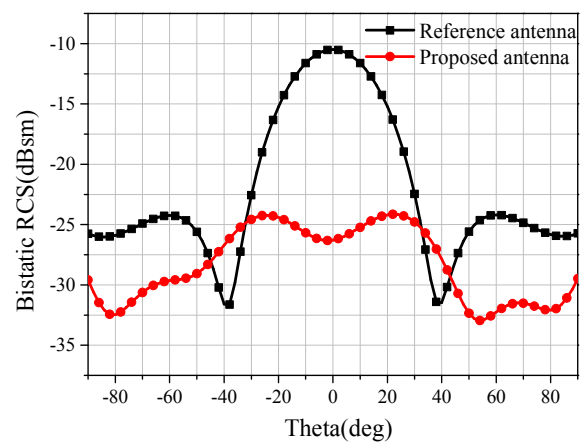

(b)

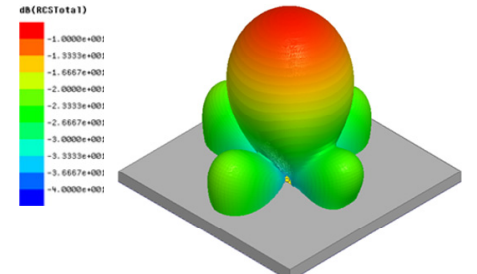

(c)

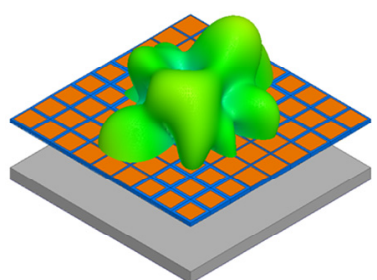

(d)
Fig. 12. Simulated bistatic RCS patterns: (a) xoz plane, (b) yoz plane, (c) 3-D pattern of the reference antenna, (d) 3-D pattern of the proposed FPRA.

\section{Fabrication and Measurement}

The photograph of the proposed antenna is shown in Fig. 13. It was tested in the anechoic to validate the simulated results. The antenna radiation patterns at $10 \mathrm{GHz}$ with and without SAMS are shown in Fig. 14. It can be seen that after loading the SAMS, the proposed antenna gain in- 
creases to $9.5 \mathrm{dBi}$ while the original antenna gain is $4.9 \mathrm{dBi}$. Figure 15 shows the measured monostatic RCS result of the proposed FPRA, from $4 \mathrm{GHz}$ to $12 \mathrm{GHz}$, the RCS is reduced obviously, which is approximately the same as the simulated one. Through simulated and measured results, we can verify the proposed FPRA has gain enhancement and low scattering properties.

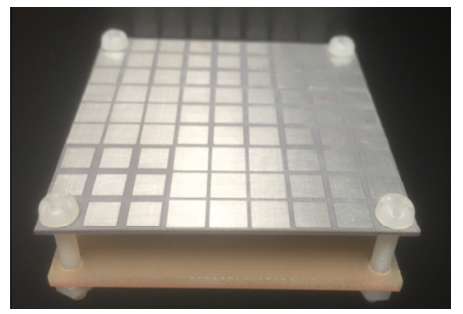

Fig. 13. Photograph of the proposed FPRA.

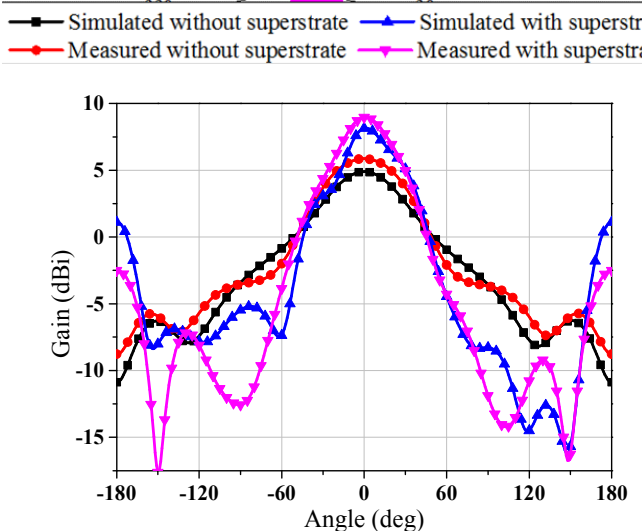

(a)

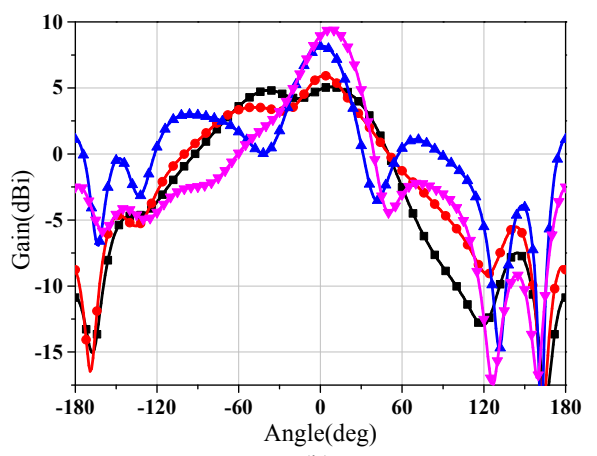

(b)

Fig. 14. Simulated and measured radiation patterns at $10 \mathrm{GHz}$ : (a) xoz plane, (b) yoz plane.

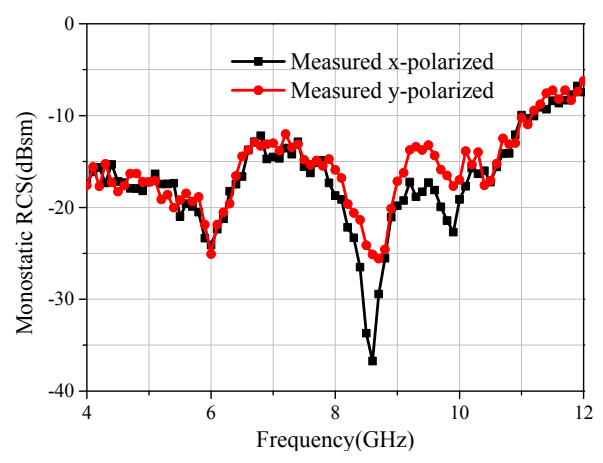

Fig. 15. Measured monostatic RCS of the proposed FPRA.

\section{Conclusion}

In this paper, a wideband high-gain and low scattering antenna using SAMS has been proposed. We presented a novel method to broaden the antenna gain enhancement bandwidth by a shared-aperture PRS, using phase compensation properties of three different sizes of two-layer FSSs. After loading the SAMS, the gain enhancement bandwidth has reached to $42 \%$ from $7.9 \mathrm{GHz}$ to $12.1 \mathrm{GHz}$ with a maximum of $7 \mathrm{~dB}$. Meanwhile, through adjusting the loading height of SAMS, the antenna RCS is reduced obviously owing to phase cancellation phenomenon. From $4 \mathrm{GHz}$ to $12 \mathrm{GHz}$, the RCS is reduced with a maximum of $25.4 \mathrm{~dB}$ at $8.6 \mathrm{GHz}$ for $\mathrm{x}$-polarized incident wave and $15.8 \mathrm{~dB}$ for $y$-polarized incident wave. Simulated and measured results are in a good agreement with each other, verifying the SAMS can improve the antenna radiation and scattering performances simultaneously.

\section{Acknowledgments}

This work is supported by the National Natural Science Foundation of China under Grant (No.61471389, No. 61501494, No. 61671464), in part by the Natural Science Foundational Research Fund of Shaanxi Province under Grant No.2017JM6025, in part by the Young Talent Fund of University Association for Science and Technology in Shaanxi, China (No.20170107). Authors also thank the reviewers for their valuable comments.

\section{References}

[1] JIA, Y., LIU, Y., GONG, S. Slot-coupled broadband patch antenna. Electronics Letters, 2015, vol. 51, no. 6, p. 445-447. DOI: 10.1049/el.2014.3905

[2] LI, S., CAO, X., XU, L., et al. Ultra-broadband reflective metamaterial with RCS reduction based on polarization convertor, information entropy theory and genetic optimization algorithm. Scientific Reports, 2016, vol. 6, p. 37409. DOI: 10.1038/srep37409

[3] MONTI, M., SORIC, J., BARBUTO, M., et al. Mantle cloaking for co-site radio-frequency antennas. Applied Physics Letters, 2016 , vol. 108, p. 113502. DOI: 10.1063/1.4944042

[4] RODES, E., DIBLANC, M., AMAUD, E., et al. Dual-band EBG resonator antenna using a single-layer FSS. IEEE Antennas and Wireless Propagation Letters, 2007, vol. 6, p. 368-371. DOI: 10.1109/LAWP.2007.902808

[5] FERESIDIS, A., VARDAXOGLOU, J. High gain planar antenna using optimized partially reflective surfaces. IEE Proceedings: Microwaves, Antennas and Propagation, 2001, vol. 148, no. 6, p. 345-350. DOI: 10.1049/ip-map:20010828

[6] HAN, Z., SONG, W., SHENG, X. Gain enhancement and RCS reduction for patch antenna by using polarization-dependent EBG surface. IEEE Antennas and Wireless Propagation Letters, 2017, vol. 16, p. 1631-1634. DOI: 10.1109/LAWP.2017.2658195

[7] SCHURIF, D., MOCK, J. J., JUSTICE, B. J., et al. Metamaterial electromagnetic cloak at microwave frequencies. Science, 2006, vol. 314, p. 977-980. DOI: $10.1126 /$ science. 1133628 
[8] ZHANG, C., CAO, X., GAO, J. Low RCS and broadband ME dipole antenna loading artificial magnetic conductor structures. Radioengineering, 2017, vol. 26, no. 1, p. 38-44. DOI: 10.13164/re.2017.0038

[9] WU, Q., PAN, P., MENG, F., et al. A novel flat lens horn antenna designed based on zero refraction principle of metamaterials. Applied Physics A, 2007, vol. 87, no. 2, p. 151-156. DOI: 10.1007/s00339-006-3820-9

[10] RAMACCIA, D., BARBUTO, M., MONTI, A., et al. Exploiting intrinsic dispersion of metamaterials for designing broadband aperture antennas: theory and experimental verification. IEEE Transactions on Antennas and Propagation, 2016, vol. 64, no. 3, p. 1141-1146. DOI: 10.1109/TAP.2016.2521871

[11] YANG, F., AMINIAN, A., RAHMAT-SAMII, Y. A novel surface-wave antenna design using a thin periodically loaded ground plane. Microwave and Optical Technology Letters, 2005, vol. 47 , no. 3 , p. $240-245$. DOI: $10.1002 /$ mop. 21136

[12] ZEB, B., GE, Y., ESSELLE, K., et al. A simple dual-band electromagnetic band gap resonator antenna based on inverted reflection phase gradient. IEEE Transactions on Antennas and Propagation, 2012, vol. 60, no. 10, p. 4522-4529. DOI: 10.1109/TAP.2012.2207331

[13] TRENTINI, G. V. Partially reflecting sheet arrays. IRE Transactions on Antennas and Propagation, 1956, vol. 4, no. 4 , p. 666-671. DOI: 10.1109/TAP.1956.1144455

[14] ZHENG, Y., GAO, J., CAO, X., et al. Wideband RCS reduction and gain enhancement microstrip antenna using chessboard superstrate. Microwave and Optical Technology Letters, 2015, vol. 57, no. 7, p. 1738-1742. DOI: 10.1002/mop.29167

[15] ZHANG, L., WAN, L., LIU, S., et al. Realization of low scattering for a high-gain Fabry-Perot antenna using coding metasurface. IEEE Transactions on Antennas and Propagation, 2017, vol. 65 no. 7, p. 3374-3383. DOI: 10.1109/TAP.2017.2700874

[16] JIANG, H., XUE, Z., LI, W., et al. Low RCS high-gain partially reflecting surface antenna with metamaterial ground plane. IEEE Transactions on Antennas and Propagation, 2016, vol. 64, no. 9, p. 4127-4132. DOI:10.1109/TAP.2016.2589964

[17] GE, Y., ESSELLE, K., BIRD, T., et al. The use of simple thin partially reflective surfaces with positive reflection phase gradients to design wideband, low-profile EBG resonator antennas. IEEE Transactions on Antennas and Propagation, 2012, vol. 60, no. 2, p. 743-750. DOI: 10.1109/TAP.2011.2173113

[18] WANG, N., LIU, Q., WU, C., et al. Wideband Fabry-Perot resonator antenna with two complementary FSS layers. IEEE Transactions on Antennas and Propagation, 2014, vol. 62, no. 5, p. 2463-2471. DOI: 10.1109/TAP.2014.2308533
[19] WEILY, A., ESSELlE, K., BIRD, T., et al. Dual resonator 1-D antenna with slot array feed for improved radiation bandwidth. IET Microwaves Antennas Propagation, 2007, vol. 1, no. 1, p. 198-203. DOI: 10.1049/iet-map:20050314

\section{About the Authors...}

Chen ZHANG was born in Shannxi. He received his M.S. degree from the Air Force Engineering University (AFEU) in 2014. He currently works towards his Ph.D. degree. In his research, he specializes in Artificial Magnetic Conductor, antenna design and RCS reduction techniques.

Xiang-yu CAO received her M.S. degree from the Air Force Missile Institute in 1989. In the same year, she joined the Air Force Missile Institute. She received her Ph.D. degree in the Missile Institute of AFEU in 1999. From 1999 to 2002, she was engaged in postdoctoral research in Xidian University, China. She was a Senior Research Associate in the Dept. of Electronic Engineering, City University of Hong Kong from June 2002 to Dec. 2003. She is currently a professor and a senior member of IEEE. Her research interests include computational electromagnetic, electromagnetic metamaterials and their antenna applications.

Jun GAO received the B.Sc and M.A.Sc degrees from the Air Force Missile Institute in 1984 and 1987, respectively. He joined the Air Force Missile Institute in 1987 as an assistant teacher. He became an associate professor in 2000. $\mathrm{He}$ is currently a professor of the Information and Navigation College, Air Force Engineering University of CPLA. He has authored and coauthored more than 100 technical journal articles and conference papers, and holds one China soft patent. His research interests include smart antennas, electromagnetic metamaterials and their antenna applications.

Si-jia LI received his M.S. and Ph.D. degrees from the Information and Navigation Institute, AFEU, in 2012 and 2016 , respectively. He is currently engaged in postdoctoral research with the same University. His research interests are electromagnetic metamaterials and their antenna applications. 